\title{
Tracking Community Naloxone Dispensing: Part of a Strategy to Reduce Overdose Deaths
}

\author{
Jennifer Dolatshahi, Lara Maldjian, Alice Welch, Casey Fulmer, Emily Winkelstein
}

Bureau of Alcohol and Drug Use Prevention, Care \& Treatment, New York City Department of Health and Mental Hygiene, Long Island City, New York, United States

\section{Objective}

Describe the development of an individual-level tracking system for community-based naloxone dispensing as part of New York City's (NYC) comprehensive plan to reduce overdose deaths. We present data from the first year of the initiative to illustrate results of the tracking system and describe the potential impact on naloxone dispensing program.

\section{Introduction}

The number of unintentional overdose deaths in New York City (NYC) has increased for seven consecutive years. In 2017, there were 1,487 unintentional drug overdose deaths in NYC. Over $80 \%$ of these deaths involved an opioid, including heroin, fentanyl, and prescription pain relievers [1]. As part of a comprehensive strategy to reduce overdose mortality in NYC, the NYC Department of Health and Mental Hygiene's (DOHMH) Overdose Education and Naloxone Distribution (OEND) Program makes naloxone kits available to laypeople free-of-charge through registered Opioid Overdose Prevention Programs (OOPPs).

Naloxone kits contain two doses of naloxone and educational materials. The OEND Program distributes kits to registered OOPPs, which then dispense kits to individuals via community-based trainings. In this context, distribution refers to kits shipped to programs, whereas dispensing refers to kits given to individuals. Increased NYC funding has enabled recruitment of more OOPPsincluding syringe exchange programs, public safety agencies, shelters, drug treatment programs, health care facilities, and other community-based programs - and greater dispensing of naloxone kits to laypeople. Naloxone distribution has undergone a dramatic expansion, from 2,500 kits in 2009 to 61,706 kits in 2017 [2]. In 2018, DOHMH aims to distribute more than 100,000 kits to OOPPs.

In order to target naloxone dispensing to neighborhoods in NYC with the highest overdose burden, we developed a tracking system able to capture individual-level geographic data about naloxone kit recipients. Prior to the development of the tracking system, DOHMH collected quarterly, aggregate-level naloxone dispensing data from OOPPs. These data included only the OOPPs' ZIP Codes but not recipient residence. OOPP ZIP Code was used as a proxy for kits dispensed to individuals. Without individual-level geographic information, however, we could not determine whether naloxone kit dispensing reached people in neighborhoods with high overdose mortality rates. To overcome these barriers, DOHMH developed a comprehensive but flexible individual-level data collection method.

\section{Methods}

To both capture individual-level data from each naloxone recipient in NYC and meet the needs of OOPPs' varying capacities, dispensing settings, and any existing organizational data requirements, DOHMH devised a two-pronged data collection system. The Naloxone Recipient Form (NRF) system, launched January 1, 2018, primarily employs a short paper form (or NRF) to collect dispensing data. The NRF is a one-page document designed with the OpenText ${ }^{\mathrm{TM}}$ TeleForm processing application. It captures individual data and OOPP information. Individual data include: reason for obtaining a kit, whether first-time receipt of a kit, age, and ZIP Code of residence. OOPP information includes: program name and ZIP Code of dispensing location. Forms are completed by OOPPs and recipients at OEND trainings, compiled by the OOPP, then scanned back to DOHMH. We then import forms into TeleForm, which reads the NRF data directly into a database without need for manual data entry and only moderate need for data verification. The second component of the NRF system allows larger organizations and dispensers in clinical settings with electronic health records to submit data extracts to DOHMH that are pulled directly from organizations' data systems. Together with these organizations, we customized these data extracts for direct importation into the master NRF database. 
To demonstrate improvements in our tracking of naloxone dispensing after the development of the NRF system, we mapped the geographic spread of naloxone kits in NYC during the first three months of 2018 (Q1 2018) by recipients' ZIP Code of residence and OOPPs' ZIP Codes.

\section{Results}

A total of 138 OOPPs [2] reported any dispensing from January to June, 2018, of which 107 reported individual-level data using the NRF system, accounting for 27,899 kits dispensed to 23,610 individual recipients [3]. Logistical barriers to implementing the NRF system varied among OOPPs, thus the data underestimate citywide dispensing during this time period. Some OOPPs experienced delays in reporting recipient-level data until a more-tailored data collection strategy was devised. Visual inspection of OOPP-level distribution and individual-level dispensing maps using Q1 2018 data (See Figure 1 and Figure 2) demonstrate the difference between OOPP-level distribution data and individual-level dispensing data. Mapping data indicate that the largest numbers of naloxone kits were dispensed to people in neighborhoods with the highest burden of overdose in NYC.

\section{Conclusions}

The NRF system provides DOHMH, as well as OOPPs in NYC, with individual-level data to more accurately track naloxone dispensing citywide. The simplicity and flexibility of the NRF system allows for timely and geographically precise data collection from dozens of organizations across NYC with little or no additional cost to OOPPs. As new organizations have registered as OOPPs, particularly large health care or human services systems, DOHMH has developed new methods for incorporating dispensing data into the NRF system. Ongoing communication with OOPPs of all types and an iterative data collection improvement process have ensured that the system remains comprehensive while also being responsive to individual program's capacities and data needs.

Our analysis comparing OOPP-level and individual-level dispensing confirmed higher numbers of naloxone kit dispensing in neighborhoods with larger numbers of overdoses, a program goal. The NRF system also provides an opportunity to target increases in naloxone dispensing to improve the effectiveness of our overdose prevention efforts.

\section{References}

1. Nolan ML, Tuazon E, Blachman-Forshay J, Paone D. Unintentional Drug Poisoning (Overdose) Deaths in New York City, 2000-2017. New York City Department of Health and Mental Hygiene: Epi Data Brief (104); September 2018.

2. NYC DOHMH Opioid Overdose Prevention Program (OOPP) Database. All data is provisional.

3. NYC DOHMH Naloxone Recipient Form (NRF) Database. All data is provisional. 
Figure 1. Number of naloxone kits distributed to OOPPs by program shipping address, Jan - Mar 2018 ${ }^{1,2}$

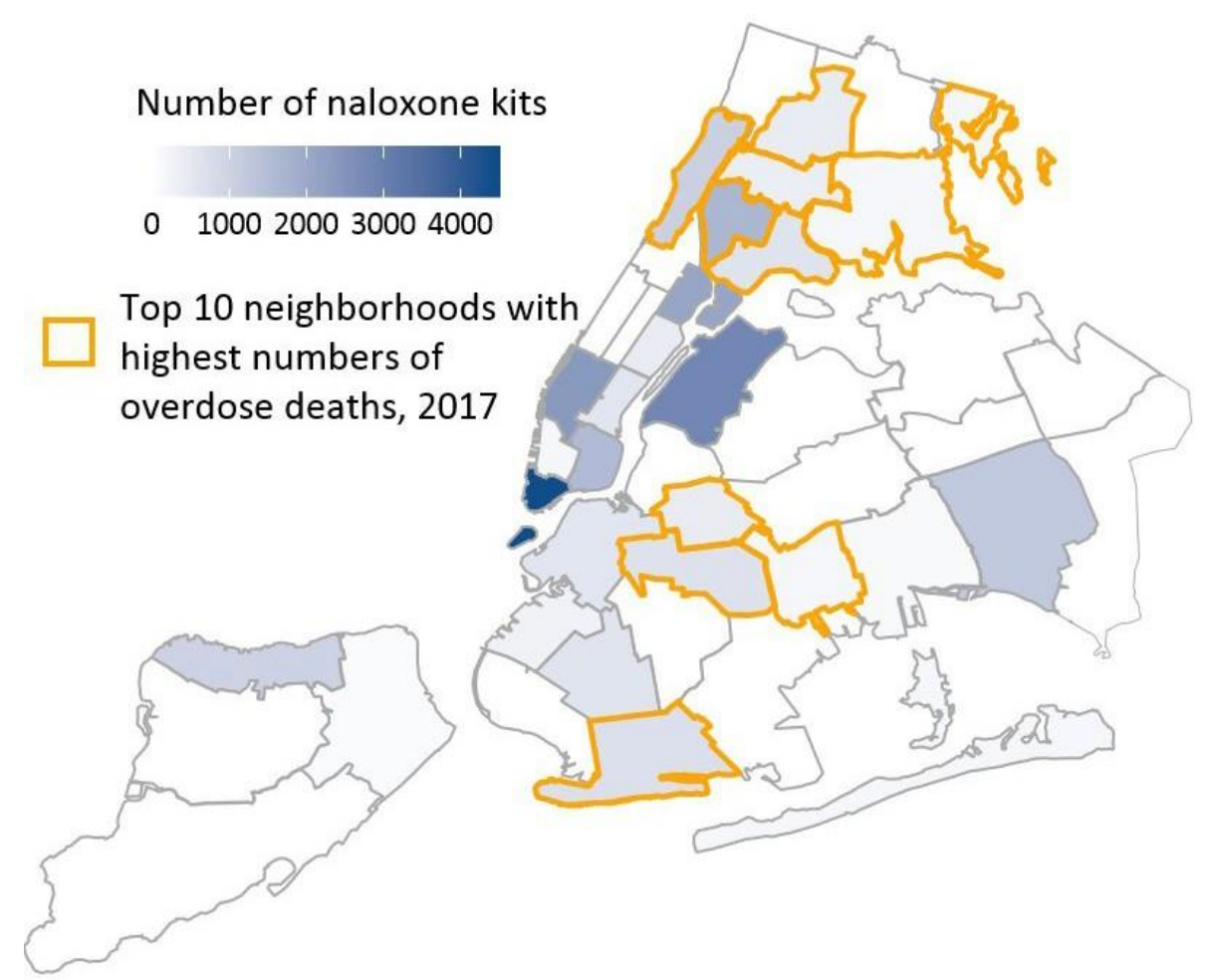

Figure 1. New York City kit distribution data (Fig. 1) did not reflect where kits were going 


\section{Figure 2. Number of people receiving naloxone kits, by residence of recipient, Jan - Mar 2018}

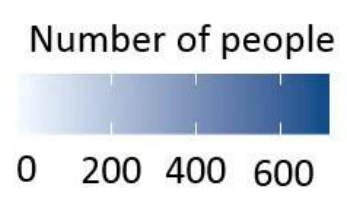

Top 10 neighborhoods with highest numbers of overdose deaths, 2017
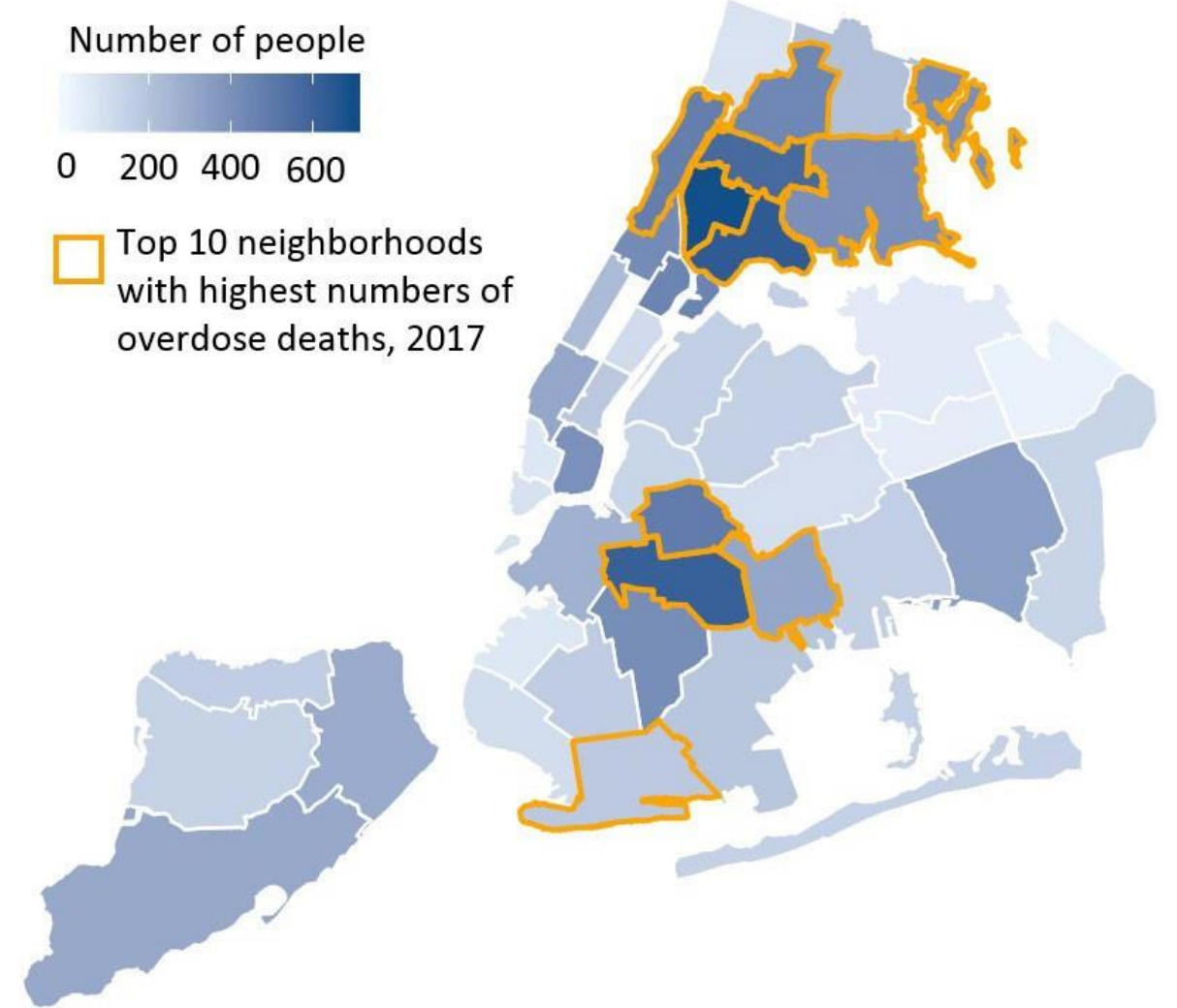

Figure 2. A new system was needed to understand naloxone dispensing. 